\title{
How many Cochrane reviews are needed to cover existing evidence on the effects of healthcare interventions?
}

Clinicians are faced with the challenge of keeping abreast of the rapidly growing clinical literature. One method that can help clinicians meet this challenge is the provision of synthesised evidence by such groups as the Cochrane Collaboration. The Cochrane Library contains the full text of Cochrane reviews, which bring together information on controlled studies (mostly randomised controlled trials) in a standard format. The first 1000 reviews were prepared by early 2001, and there are now more than 1600 available. ${ }^{1}$ The task of synthesising the clinical literature is a huge undertaking, and in this editorial we will use the number of studies in a typical Cochrane review to provide an estimate of the number of Cochrane reviews that are needed to cover all trials currently in the Cochrane Central Register of Controlled Trials (CENTRAL)..$^{2-3}$

CENTRAL is the main source used by Cochrane reviewers to locate studies. It contains citations to more than 300000 reports of studies, which may be eligible for inclusion in Cochrane reviews. CENTRAL is reference-based rather than study-based, and therefore multiple references may be included for a single trial.

We examined 1000 reviews from the Cochrane Database of Systematic Reviews in Issue 1, 2001, of the Cochrane Library to determine the average number of studies, and references to the studies, included in a typical Cochrane review. Comparing this with an estimate for the number of relevant references in CENTRAL, it is possible to predict how many more Cochrane reviews are needed to cover the healthcare research already in CENTRAL.

Of the 1000 reviews, 11 had been replaced by notices of withdrawal, leaving 989 complete reviews. The included studies in these reviews were hand counted by 1 author (SM), and this process was confirmed by a computerised count of the database files. We used median values to express averages because the small number of reviews with unusually high numbers of studies would lead to a positive skewing of mean values. We used a random sample of 104 reviews to obtain the average number of references per study in a Cochrane review.

The 989 Cochrane reviews included information on a total of 9778 studies. The number of studies per review ranged from $0-136$, with a median of 6 (interquartile range 3 to 12). 48 reviews $(5 \%)$ did not include data from any studies, and 19 reviews $(2 \%)$ included $\geq 50$ studies. In the 104 reviews where the number of references for each included study was counted, 1051 studies were reported in 1514 references. The median number of references per study per review was 1.1 (interquartile range $1.0-1.5)$.

An average of 1.1 references per study suggests that 300000 references in CENTRAL might refer to about 270000 studies. Thus, if the average Cochrane review includes 6 studies, 45000 Cochrane reviews would be needed to cover 300000 references. However, the 1.1 references per study might be an underestimate and some of the records in CENTRAL might not relate to studies that are suitable for Cochrane reviews. For example, the Cochrane Stroke Group reported an average of 2.1 references per study in their Specialized Register of Stroke Trials (4525 reports corresponding to 2132 stroke trials). ${ }^{4}$ On the basis of the Stroke Group figure, the estimated number of additional Cochrane reviews needed to cover all references in CENTRAL would be 24000 . However, this still does not take into account the probability that some references in CENTRAL would not be eligible for Cochrane reviews.

Comparing the typical size of one of the first 1000 Cochrane reviews with that found by descriptive studies of other types of systematic reviews is problematic. Such studies have found averages of 10 or 14 studies per review. ${ }^{5}$ Instead of trying to estimate the appropriate size of an average systematic review, our finding of the typical size of a Cochrane review based on the first 1000 reviews combined with information on the content of CENTRAL can provide a reasonable estimate of how much work remains to be done.

Even with the assumption that future Cochrane reviews will each include about 6 studies, the above estimates of 24000 or 45000 additional reviews are likely to be excessive, because not all the references in CENTRAL (or the studies they report) might be suitable for inclusion in new Cochrane reviews. For example, there will be records for references to studies that are already included in Cochrane reviews but for which the reference is not yet linked appropriately to the study in the review, and some references appear more than once in CENTRAL. Some of the studies will relate to interventions that are no longer used or will only have information that is not relevant to healthcare decisions, and some references do not relate to studies of healthcare interventions. Finally, there will be some references to studies that are eligible for existing Cochrane reviews but have not yet been included.

Therefore, if 45000 or 24000 are accepted as overestimates, some correction is needed. At the moment, no data exist to help with this estimation, but we think it is reasonable to predict that at least 10000 Cochrane reviews are needed to cover a substantial proportion of the studies relevant to health care that have already been identified, taking into account the typical size of a Cochrane review, number of included studies and their references, and the probable number of eligible studies in CENTRAL. This estimation is a crude approximation but is probably a reasonable minimum. Reviews will also be needed on the many thousands of new studies that are published each year, and the 10000 reviews will need to be kept up to date.

When will 10000 reviews be ready? The Cochrane Database of Systematic Reviews is updated every quarter to include new Cochrane reviews, as well as those that have been updated. Currently, there are more than 1600 Cochrane reviews and 1200 protocols for reviews in progress in the Cochrane Database of Systematic Reviews. ${ }^{1}$ The figure shows the recent growth in the number of reviews. Fitting a simple model to the data allows us to predict when this growth will reach 10000 Cochrane reviews. We estimate that this will be between 2010 and 2015, and we look forward to either being proved correct or pessimistic in our estimate. If, however, 24000 or 45000 reviews are needed to cover all existing randomised trials of health care, it will take 


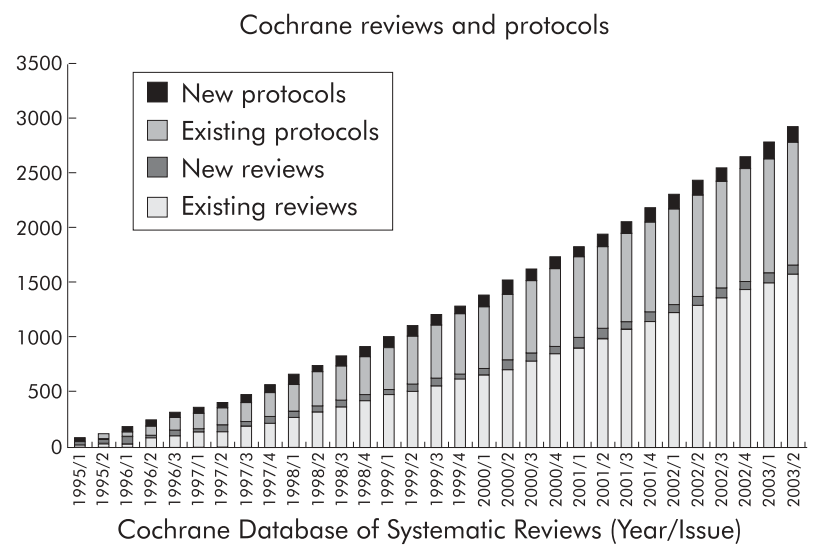

until about the 2030s and 2040s, respectively, if the current rate of growth continues.

The need to produce and use reviews of healthcare interventions should also be faced by trialists. If reports of new research were routinely discussed within the context of updated systematic reviews, users of this new research would, quite correctly, be presented immediately with a key part of the evidence needed to make decisions about health care ${ }^{6}$

The implications of our study represent considerable challenges not only to the Cochrane Collaboration and others involved in the production of systematic reviews, but also to organisations involved in policy decisions about health care that aim to base treatment guidelines and guidance on up to date reviews of healthcare evidence. The challenges to be addressed in producing 10000 systematic reviews and keeping them up to date centre on the engagement of enthusiasm, skills, time, and resources to undertake and support such work. The alternatives, however, might be to rely on non-systematic, out of date summaries of evidence supplemented by an occasional systematic review when it is available, or to make decisions without concern for reliable evidence on the likely effects of the interventions used. ${ }^{7}$ The authors would be very pleased to be contacted by anyone wishing to contribute to growth in the number of Cochrane reviews.

Conflict of interest statements for authors: Susan Mallett was employed during this project as a Research Assistant at the UK Cochrane Centre. Mike Clarke is employed as Director of the UK Cochrane Centre.

\section{Acknowledgments}

We thank Iain Chalmers, Carol Lefebvre, Sally Hopewell, and Peter Langhorne for comments on preliminary versions of this manuscript. We thank Mark Starr of Update Software for providing computer counts of included studies.

This work was done as part of the programme of methodological research being undertaken at the UK Cochrane Centre, which is funded by the UK National Health Service Research and Development Programme.

Disclaimer: The views expressed in this paper represent those of the authors and are not necessarily the views or the official policy of the Cochrane Collaboration.

\section{SUSAN MALLETT, DPhil MIKE CLARKE, DPhil UK Cochrane Centre Oxford, UK}

1 The Cochrane Database of Systematic Reviews. In: The Cochrane Library, Issue 2, 2003. Oxford: Update Software.

Issue 2, 2003. Oxford: Up a sy Softwac kever. The Cochrane Central Register of Controlled Trials (CEN Cochrane Library, Issue 2, 2003. Oxford: Update Software.

3 Dickersin K, Manheimer E, Wieland S, et al. Development of the Cochrane Collaboration's CENTRAL Register of controlled clinical trials. Eval Health Prof 2002;25:38-64.

4 Thomas B, McInnes A Sandercock P. Is it worth searching for reports of stroke trials published in languages other than English? 9th International Cochrane Colloquium, Lyon, France, 9-13 October, 2001.

5 Jadad AR, Cook DJ, Jones A, et al. Methodology and reports of systematic reviews and meta-analyses: a comparison of Cochrane reviews with articles published in paper-based journals. JAMA 1998;280:278-80.

6 Clarke M, Alderson P, Chalmers I. Discussion sections in reports of controlled trials published in general medical journals. JAMA 2002;287:2799-801.

7 Oxman A. The Cochrane Collaboration in the 21st century: ten challenges and one reason why they must be met. In: Egger M, Davey Smith G, Altman DG, editors. $2 \mathrm{~d}$ ed. Systematic reviews in health care: meta-analysis in context. London: BMJ Publishing Group, 2001

\section{How to cite material from Evidence-Based Medicine}

Citation of material from the Notebook

Milne R, Hicks N. Evidence-based purchasing [EBM Note]. Evidence-Based Medicine 1996 May-Jun;1:101-2.

Citation for material taken from a structured abstract, written without attribution by a staff member

Antihypertensive drugs decrease mortality, coronary events, and stroke in elderly persons [abstract]. Evidence-Based Medicine 1996 May-Jun;4:105. Abstract of: Pearce KA, Furberg CD, Rushing J. Does antihypertensive treatment of the elderly prevent cardiovascular events or prolong life? A meta-analysis of hypertension treatment trials. Arch Fam Med 1995;4:943-50.

Citation for material taken from a commentary to an article

Olds D. Commentary on "Home visiting programmes reduce childhood injury" Evidence-Based Medicine 1996 May-Jun;4:112. Comment on: Roberts I, Kramer MS, Suissa S. Does home visiting prevent childhood injury? A systematic review of randomised controlled trials. BMJ 1996;312:29-33. 